\title{
Adaptive Knowledge Visualization Systems: A Proposal and Implementation
}

\author{
Xiaoyan Bai, David White, and David Sundaram
}

\begin{abstract}
Visualizations are crucial to the creation, transfer and sharing of knowledge. However, there are three essential problems associated with existing knowledge management systems (KMS) and their visualizations. First, KMS tend to provide weak support for leveraging visualization to accomplish transformation, discovery and learning of knowledge. Second, KMS do not possess mechanisms to aid in sensing, analyzing and responding to contextual changes. Third, KMS are weak in adapting to diverse and changing problem and knowledge worker contexts. To address these problems, we propose and implement a contextual adaptive knowledge visualization model and system in this paper. Furthermore, we demonstrate the system implementation through a sequence of scenario-driven illustrations.
\end{abstract}

Index Terms - knowledge visualization, context, knowledge worker, adaptive, knowledge creation, knowledge sharing, model, implementation.

\section{INTRODUCTION}

Visualizations, as an essential component of knowledge management systems (KMS), are integral for creating, transferring and sharing knowledge. Knowledge visualization is playing an increasingly crucial role in knowledge management and the design and development of knowledge management systems $[1,2,3,4,5,6,7,8]$. With the rapid advances achieved in the field of information visualization, visualizations nowadays can be applied to a wide range of contexts where knowledge workers need to explore, create, represent, present, transfer and/or share knowledge.

Contexts may be articulated in different ways depending on the underlying research domains, for example, [9], [10] and [11]. In the domain of knowledge management, we define knowledge visualization context as the information of any environmental entities that influences knowledge visualization design, implementation, application/use, and evaluation. Such contextual information is associated with the decisional problem context where knowledge visualizations are deployed, such as relevant problem situations, time, space, social context, and technological context. It also incorporates the visualization profiles of knowledge workers such as their cognitive styles/abilities, purposes, personal characteristics and preferences, prior knowledge, age and gender.

Manuscript receive August 10, 2011; revised August 25, 2011.

Xiaoyan Bai, David White, and David Sundaram are with Department of Information Systems and Operations Management, University of Auckland, Auckland, New Zealand.
Knowledge visualization context is complex and dynamic in nature. Such complexity is caused by visualization problem and stakeholder contexts involving extensive factors that are diverse and constantly changing. The problem context of the same knowledge worker may also vary over time. As the knowledge worker becomes familiar with the underlying visualization tools and decision problems, his/her visualization profile (e.g. cognitive characteristics, personal preferences, prior knowledge, etc.) may also change accordingly. Contextual changes often lead to dynamic and changing visualization requirements. It can even make the visualizations in use become ineffective and/or irrelevant to the new problem and/or knowledge worker context.

To address the above contextual complexities, visualization environments embedded in and/or designed to support KMS should be able to not only sense, analyze and respond to the changes in the visualization contexts but also mediate between the problem and the knowledge workers through the explicit provision of action and presentation languages. More specifically, they need to provide flexible support for (1) creating/manipulating/transforming/ improving/disposing visualization solutions and (2) maintaining the effectiveness of the solutions under the changing/evolving knowledge visualization context. For example, they should enable the knowledge workers to adapt/personalize/customize the visualizations as to better suit their requirements at different learning or skill development stages from novice to master.

However, existing knowledge management systems and their visualizations tend to provide weak support for the above requirements. By reviewing and synthesizing the literature on knowledge management and knowledge management systems, we identified three essential problems. Firstly, there is a lack of support for using visualization in KMS to accomplish the transformation, discovery and learning of knowledge. KMS tend to offer reasonable support for the initial stages involved in the knowledge discovery process [12] such as data extraction, cleansing, integration and simple transformations but provide weak support for the subsequent transformation, discovery and learning stages. Secondly, many KMS have a concept/content/case/rule orientation. They are excellent at being repositories of data, concepts, cases, etc. and are very useful for reference. However, these systems in general do not possess mechanisms to aid in (1) the sensing of the changing problems and/or knowledge worker contexts, (2) the analysis, transformation and learning of the new contexts, and (3) responding to the contextual changes and the associated visualization requirement changes and the knowledge garnered. Thirdly, it is insufficient for KMS to merely 
generate and store expert knowledge under a particular context. Besides knowledge creation, equally important is that KMS can adapt to diverse knowledge contexts so that relevant and useful knowledge can be presented to knowledge workers in the right place at the right time so as to support various user tasks involved in information retrieval/query/discovery/explanation/ navigation and decision making.

The gap between the above requirements for visualization and the support offered by KMS motivated us to propose and implement a flexible system for better aiding knowledge creation, transfer and sharing, namely Contextual Adaptive Visualization Environment (CAVE).

In this paper, we review the most relevant literature that helped us develop the ideas and concepts of CAVE in section 2. Based on the literature review, we introduce a definition of CAVE in section 3 . We then proceed to propose a conceptual model of CAVE to illustrate the key concepts involved in CAVE in section 4. Next, we explicate how the CAVE model can guide the design and development of visualizations in knowledge management systems in section 5. To support the implementation of CAVE, we propose a CAVE architecture in section 6. To validate our proposed artefacts, we implemented a prototypical system to demonstrate how CAVE can help knowledge workers better leverage visualizations to address their problems and/or tasks under the changing and evolving knowledge visualization context. The prototype implementation is discussed in section 7 .

\section{REVIEW OF INFORMATION VISUALIZATION REFERENCE MODELS}

Information visualization reference models outline the key tasks or building blocks for representing and presenting data. Reviewing such reference models provides a good starting point for us to define and design CAVE. Typical reference models that are relevant to our study are information visualization pipeline [13], table notation framework [14], and data state model [15]. Such reference models often serve as the basis for designing and developing visualization techniques and systems [16]. For example, Santos and Brodlie [16] pointed out that NAG IRIS Explorer [17] and the visualization toolkit [18] were constructed based on the reference model proposed by Haber and McNabb [19]. The following sub-sections review the three representative reference models respectively.

\section{A. Information Visualization Pipeline}

In this visualization pipeline model, source data are presented in certain visual forms via a set of transformations, i.e. data transformation, visual mappings, and view transformation [13]. More specifically, the raw data, which may come from a variety of sources, firstly are re-organized and converted into the form of data tables, by which data can more easily satisfy the requirements of the subsequent visual encoding and presentation tasks. Then, the data tables are transformed into proper visual structures via visual mappings. The visual structures represent the source data by leveraging a series of spatial substrates, marks and their associated graphical properties. For representing the same data, there could be multiple visual mapping options to choose and the selected option should be "faster to interpret, can convey more distinctions, or leads to fewer errors" [13]. Finally, view transformations (such as location probes, viewpoint controls and distortions) are applied to visual structures so that the data can be displayed in appropriate visual formats for users to observe.

\section{B. Table Notation Framework}

The table notation framework, introduced by Card and Mackinlay [14], extracts the fundamental building blocks involved in visualization development. This framework can be applied to facilitate analyzing, comparing and understanding visualization techniques. Based on this framework, a visualization technique can be decomposed into a set of fundamental components such as marks, controlled processing graphical features, retinal encodings and positions. The framework is quite useful for identifying the differences and similarities among multiple visualization techniques. Furthermore, it also reveals a way to design and develop new visualization techniques. Card and Mackinlay [14] argued that a new visualization could be constructed by selecting and integrating a new set of fundamental components. Visualizations may be modified or customized by replacing their underlying components.

\section{Data State Model}

Building on the table notation framework [14], Chi and Riedl [15] proposed a data state model that highlights the transformation operations performed within and in between the essential data stages involved in a general information visualization process. Compared to the table notation framework and the visualization pipeline model, the data state model offers a clear view of how the intermediate data transitions are conducted within the visualization process. This model uses between-stage transformations (i.e. data transformation, visualization transformation, and visual mapping transformation) to modify the structure of source data. In contrast, the within-stage transformations (i.e. value stage operator, analytical stage operator, visualization stage operator, and view stage operator) do not change the data structure but facilitate the between-stage transformations. Similar to the table notation framework, the data state model also provides insight into how visualizations can be created, customized and modified by changing the underlying within-stage and between-stage transformations.

Building on top of visualization reference models as well as the ideas and concepts involved in context modeling and knowledge management, we propose a definition of CAVE in section 3 and a conceptual level CAVE model in section 4.

\section{Definition of ConteXtual AdAPtive KnOWLEDGE VISUALIZATION SYSTEM}

We define Contextual Adaptive Visualization Environment as a context-sensitive, adaptive platform that helps knowledge workers to continuously monitor the changing/evolving context of their problem, sense and analyze the changes in the context, and respond to the problem by utilizing data, knowledge models (problem and visual), solvers and scenarios to create and manage effective visual compositions. The key purpose of CAVE is to assist 
knowledge workers with sensing, analyzing and responding to the changes happened in the knowledge visualization context.

CAVE has three fundamental features that differentiate the system itself from other visualization systems. First, CAVE allows knowledge workers to monitor, define and model the impact from both knowledge visualization context and the related contextual changes on visualization requirements. Second, CAVE provides flexible support for knowledge workers to re-design and re-modelling of visual compositions so as to respond to the changes and requirements through appropriate re-design and re-modelling of visual compositions. Third, CAVE emphasizes the importance of the flexibility and reusability of knowledge visualization solutions. It treats a knowledge visualization solution as a combination of problem-related and visualization-related data, models, solvers and scenarios. The effectiveness of the entire solution depends on the match between the problem-related components and visualization-related components. According to [20], keeping these problem/visual components separate may optimize the system's flexibility for responding to contextual changes and their associated visualization requirement changes.

The ideas and concepts involved in CAVE are further delineated through a conceptual level CAVE model in the subsequent section.

\section{CONTEXtual AdAPtive KNOWledge Visualization MODEL}

For better elaborating on how CAVE interacts with problem and knowledge worker contexts, we propose a conceptual level sense and respond model of CAVE. The CAVE model aims to demonstrate the key building blocks of a contextual adaptive knowledge visualization system and reveal the mechanisms about how these building blocks could relate together for creating and managing context-adaptive knowledge visualizations (Fig. 1).

As illustrated in Fig. 1, the CAVE model supports sensing the problem context and responds to it through the creation and adaptation of visualization. We envisage it as a two-stage process. In the initial stage, the problem context/real world is sensed through various mechanisms such as real time feeds, databases, and human input which together contribute to CAVE understanding the problem, purpose, surrounding, state, time, tasks (knowledge and visualization), etc. This understanding by CAVE and subsequently by the knowledge worker enables them together to respond to what has been sensed through a variety of measures. The responses could be at different levels: parametric change (single loop learning), introduction/modification/deletion of variables of model (double loop learning), and/or transformational changes at a deep and broad level (triple loop learning). Triple loop learning is especially important when the context changes significantly.
Furthermore, CAVE mediates between the problem and knowledge workers through the explicit provision of action and presentation languages. A knowledge worker may engage with CAVE through a proper visual presentation language which enables him/her in understanding and sensing the contexts and responding appropriately through a visual action language. Obviously what is presented to the knowledge worker is adapted according to the knowledge worker type, profile, ability and his/her ultimate purpose(s).

This definition of CAVE model raises a set of fundamental requirements for designing and developing visualizations in knowledge management systems, which are elucidated in the following section.

\section{REQUIREMENTS OF DEVELOPING CONTEXTUAL ADAPTIVE KNOWLEDGE VISUALIZATION SYSTEM}

The conceptual CAVE model can provide guidance on how visualizations of a knowledge management system should be implemented so as to adapt to the changing and evolving knowledge visualization context. In a nutshell, there are four principal functional requirements that need to be fulfilled, that is, visual solution creation, visual solution modification/ customization/enhancement, visual solution integration, and visual solution transformation. These requirements are explicated in the following subsections.

\section{A. Visual Solution Creation}

The changes involved in knowledge visualization context often lead to the variation of visualization requirements, which may cause the existing visualizations to become completely irrelevant to the new context and requirements. In such circumstances, knowledge workers may need to build new visualization solutions so as to better address their interested problem. Accordingly, a knowledge management system should enable the knowledge workers to create new visualizations in a flexible fashion. The knowledge workers should be able to develop new visualizations either from scratch or based on existing reusable visualization components. This can be achieved through selecting and mapping appropriate CAVE problem and visualization components.

\section{B. Visual Solution Modification/Customization /Enhancement}

A knowledge visualization, which can address a particular problem/task/purpose under a specific context, may not be able to achieve the same level effectiveness when problem and knowledge worker contexts change over time and space. Therefore, a knowledge visualization system should offer the capabilities for knowledge workers to flexibly modify, customize and enhance visualizations so as to suit the changing requirements. With CAVE, visual solution modification/customization/enhancement can be performed by appropriately adjusting and/or replacing the problem and visualization components involved in the existing solution, for example, selecting the desired visual representations, changing the colour or the hue, modifying model input/output parameters, etc. 


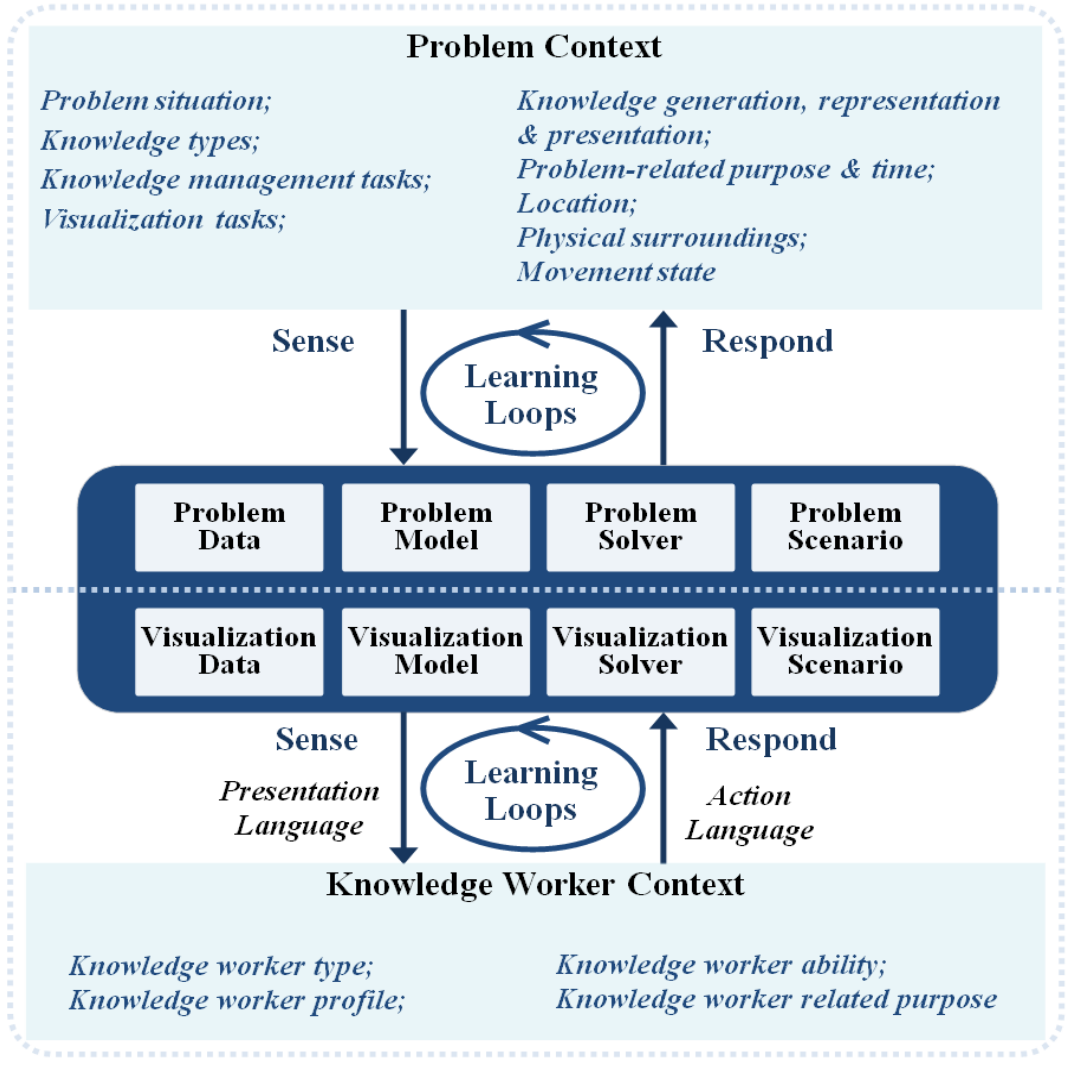

Fig. 1.A Conceptual Model of Contextual Adaptive Visualization Environment

\section{Visual Solution Integration}

This functional requirement is concerned with flexibly combining the visual output generated by different visualization techniques/solutions so as to present a rich view of the underlying data. Due to the changing and dynamic nature of knowledge visualization context, visualizations are often required to reveal knowledge discovered for understanding different features/facets of the data. However, visualization techniques have their specific focus on handling particular types of data and reflecting particular features of the source data [21]. In other words, no single visualization solution can address all data types, visualization purposes and fit in all kinds of problem and knowledge worker contexts. Therefore, integrating multiple visualization techniques/solutions becomes a natural and effective way to enable users to explore more features of the source data [22]. Visual solution integration may need to be performed against a single data source or multiple data sources.

\section{Visual Solution Transformation}

Besides creating, modifying and integrating visual solutions, visual solution transformation is equally important for maintaining the effectiveness of a solution across different problem and knowledge worker contexts. Therefore, knowledge management systems need to enable users to transform visualizations from one type to another in a flexible, seamless and efficient manner. This will enable the users to visualize the same set of data through different angles/perspectives via different visualization techniques/solutions. With CAVE, visual solution transformation can be accomplished through mapping the simple problem/visualization components into powerful visual compositions to explore/understand/solve problems that knowledge workers face.

In order to fulfil the requirements posed above and support the conceptual CAVE model, we propose a system architecture that can be applied to guide the design and implementation of CAVE in the next section.

\section{ConteXtual AdAPtiVe KnOWLEDGe Visualization ARCHITECTURE}

This three-tier system architecture aims to provide guidance for the development of a contextual adaptive knowledge visualization system. It highlights the core system components involved in CAVE and the interactions among them (Fig.2).

Knowledge visualization context should be well understood by knowledge workers. When contextual changes occur, the knowledge workers analyze these changes and identify the corresponding visualization requirements. The user interface allows the knowledge workers to communicate their requirements with the system and consume the services offered by the lower layers. 


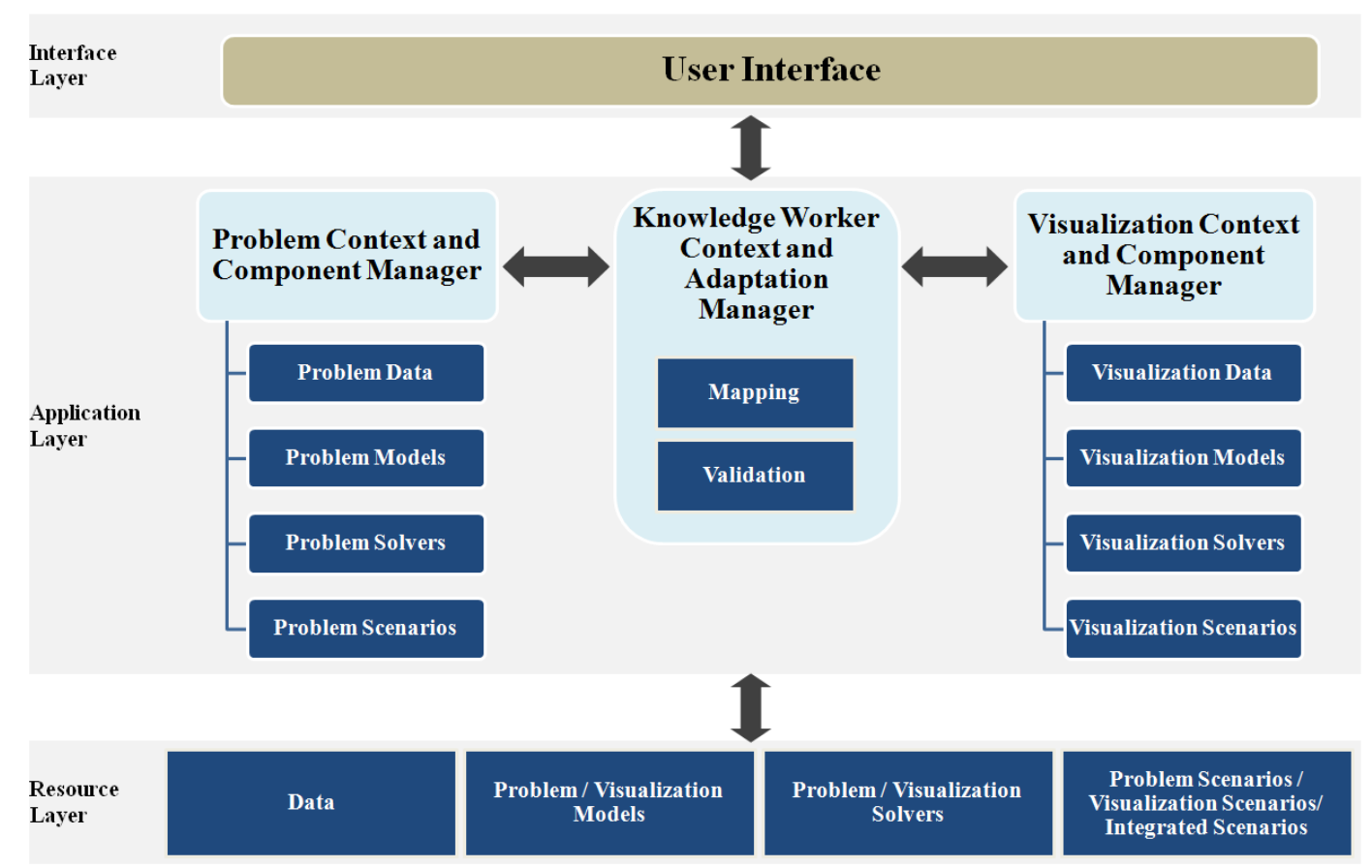

Fig. 2.CAVE Architecture

The user requirements are handled by the three key system components at the application layer, that is, problem context and component (PCC) manager, visualization context and component (VCC) manager, and knowledge worker context and adaptation (KWCA) manager. The PCC manager helps the knowledge workers create and manage problem scenarios through selecting and integrating relevant problem data, models and solvers. A problem scenario is an abstraction of the real-world problem that the knowledge workers seek to resolve, defines the way of how the source problem data and models are manipulated by the solvers, and generates useful and relevant information for the users. Such information provides the source data for visualization. When the knowledge visualization context changes, the PCC manager allows the users to adapt to the contextual change(s) through modifying and/or creating new problem scenarios that can suit the new context. The output of the PCC manager is instantiated problem scenarios which provide the input data for VCC manager to visualize. Compared to the PCC manager, the VCC manager enables the knowledge workers to apply appropriate visualization models and solvers to the input data to build visualization scenarios. Such visualization scenarios can be adapted to address new contextual changes and visualization requirements by modifying and/or replacing the involved components. The communication between the PCC and VCC managers is handled by the KWCA manager where the integrated scenarios are created and managed. The output from the integrated scenarios is presented to the users through the top tier user interface and all the problem and visualization related data, models, solvers and scenarios are kept in a persistent components storage at the bottom tier

To validate this architecture, we implemented a prototypical contextual adaptive knowledge visualization environment, which is briefly delineated in the following section.

\section{IMPLEMENTATION}

The CAVE prototype is implemented based on a multi-tier object-oriented component-based system architecture which is designed based on the CAVE model. This vertical prototypical system leverages a set of Microsoft technologies, that is, Bing map, windows presentation foundation, ADO.NET entity framework, and SQL Server. However, a similar system could be developed by utilizing equivalent technologies from other vendors such as Oracle and IBM. The prototype enables the sensing of contextual changes through accessing a number of historical and/or real-time data streams. Apart from monitoring and communicating with these data streams, the system also supports the creation of problem and visualization scenarios that enable knowledge workers to sense and become aware of emerging situations. The impact from the contextual changes is reflected by the adjustment of visualization requirements. The prototype assists the knowledge workers to respond to the contextual changes through refining or re-creating knowledge visualization solutions, e.g. mapping the problem scenario to a more appropriate visualization scenario to better fit in the new problem/knowledge worker contexts.

To help with demonstrating the support of the CAVE prototype, we introduce two cases, i.e. Napoleon's army march to Russia, and child statistics. The former case resides more in the domain of historical management while the latter is mainly about statistical data analysis. The Napoleon's march case is based on Napoleon's defeat in Russia in 1812. In June of 1812, Napoleon commenced his march on Russia from the Polish-Russian border with about 422,000 troops and retreated back to Poland in December of 1812 with only about 10,000 men alive. In this case, we focus on exploring and visualizing the relationships between army size reduction and its potential causing factors such as temperature, speed, location altitude, enemy size and available resources at each location, etc. In comparison, the child statistics case focuses 
on extracting knowledge and discovering patterns from a set of child/education related indicators and statistics in different countries, e.g. primary school completion rate, expenditure per student, literacy rate of adult, etc. These child-related statistics are quite useful for studying the characteristic of children. For example, the statistics on the distribution of NZ child population reveal the characteristics of how the child population of different ethnic groups varied in different regions every year. Both cases require visualizing spatial temporal multi-dimensional data.

The adaptation to knowledge visualization context changes may occur at different levels of abstraction. At macro level, the contextual changes involve the whole problem situation/domain changes. In contrast, at micro level, they can be associated with any changes to problem/visualization data, models and solvers and knowledge workers.

The above two cases demonstrate changes of knowledge visualization contexts at both macro level where the problem situation changes from the Napoleon's march case to the child statistics case and micro level where different knowledge workers expose different visualization preferences and requirements. By illustrating how well both cases can be supported by the CAVE prototype, we show the features/functionalities of the prototype for supporting diverse and changing knowledge visualization contexts and requirements at both macro and micro levels. The prototype distils knowledge and patterns through the design and implementation of layered, zoomable and animated problem-visualization scenarios.

\section{A. Support for Macro Level Contextual Changes}

To support macro level contextual changes, the CAVE prototype allows knowledge workers to build various problem-visualization scenarios with catering for different sets of requirements. For example, to visualize the army size changes of Napoleon's main troop along the route, we created an integrated problem-visualization scenario that uses the red line to indicate the route and pie charts to display the percentage of the survivors (Fig. 3). To assist with exploring the relationships between the army size and other invasion and retreat related information (such as temperature, army march speed and resources at different locations) of Napoleon's main troop, we built an integrated animated problem-visualization scenario as illustrated in Fig. 4 and Fig. 5. The visualization design of this scenario builds on top of the map portraying the defeat of Napoleon's army in Russia published by Charles Joseph Minard in 1869 [23]. This scenario utilizes the width of the route band to indicate the army size and demonstrates how the army size diminishes as the temperature, speed and resources change along the route in an animated fashion.

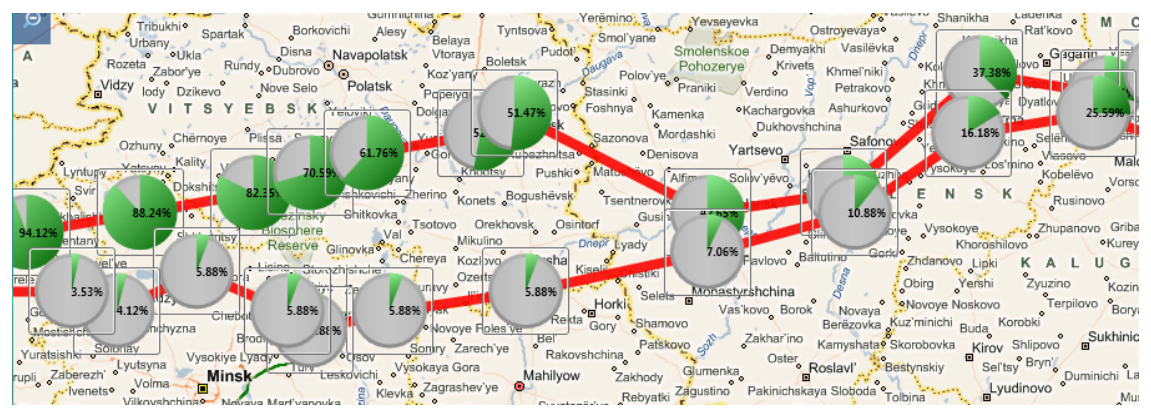

Fig. 3.A static zoomable problem-visualization scenario for discoverying patterns of army size changes

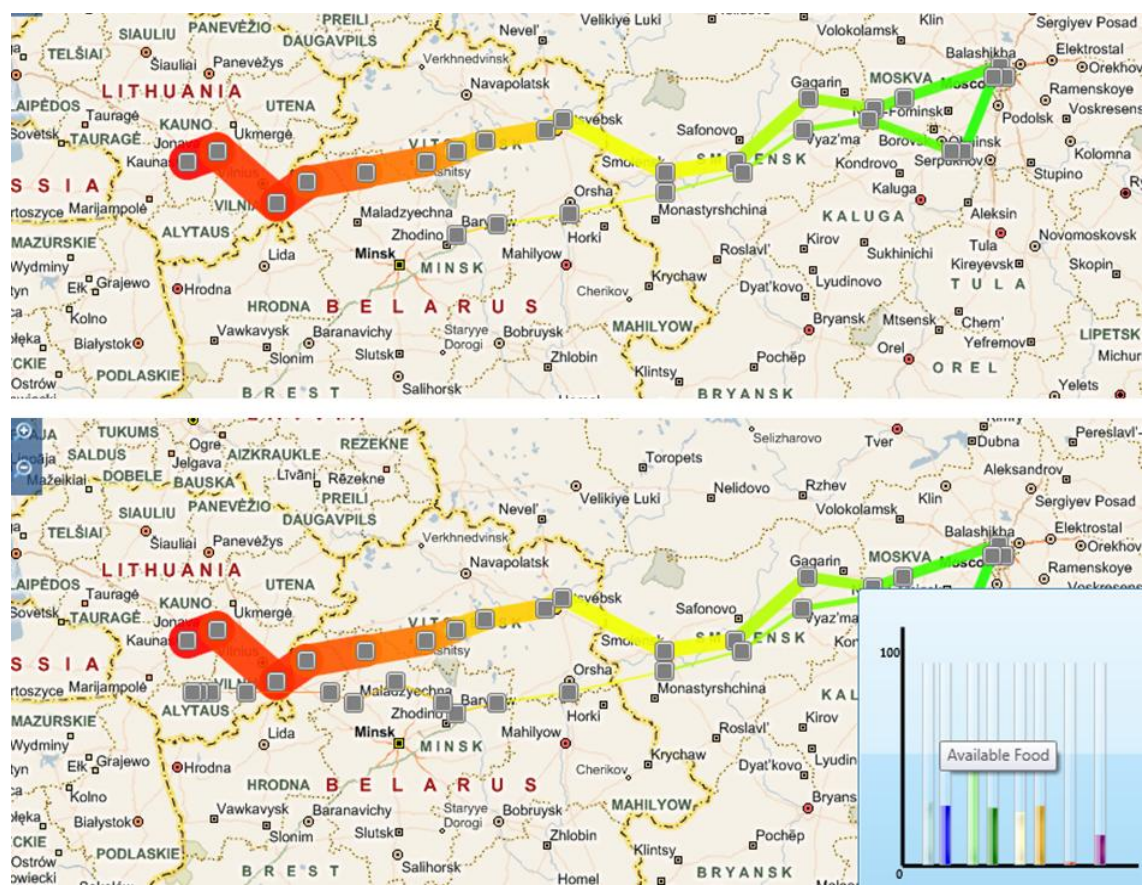

Fig. 4.An animated problem-visualization scenario for discovering relationships among army size, temperature, speed and resources 


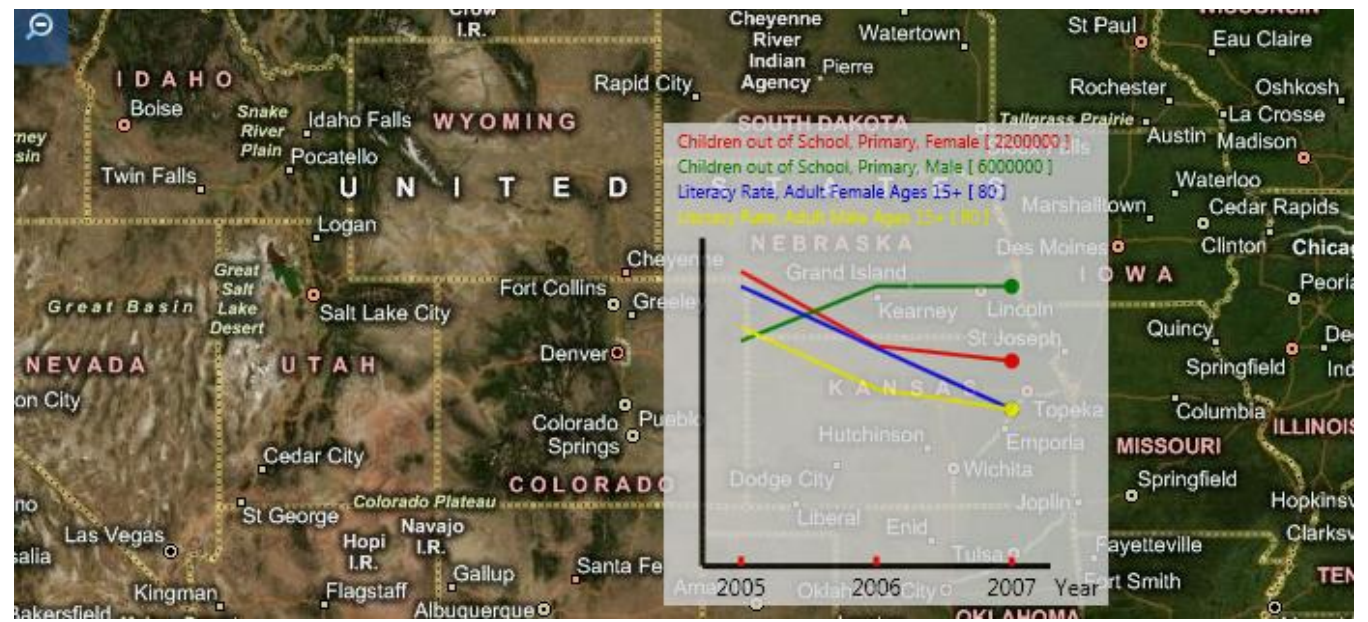

Fig. 5.A static zoomable problem-visualization scenario illustrating trends of multiple indicators in different countries

\section{B. Support for Micro Level Contextual Changes}

Knowledge sharing among different knowledge workers can require the system to accommodate their diverse visualization requirements of them. For example, some knowledge workers may prefer to use colour to present a high level overview of the child/education indicators to help with their comprehension of the knowledge. In contrast, others may like to watch and/or listen to the related media bites of the child/education indicators through vivid video/audio files. An example of a three-layer integrated problem-visualization scenario is demonstrated through Figures 6-8.

Fig. 6 shows four indicators for each country, i.e. female children out of primary school, male children out of primary school, literacy rate of female adults, and literacy rate of male adults. These indicators are represented by the following colours, i.e. red, green, blue, and yellow, in respective. For each indicator, deeper colours indicate higher values and lighter colours mean lower values. By zooming into a detailed level, the information about how the four indicators vary across consecutive years in different countries is presented in line graphs in Fig. 7. The comparison among indicators enables knowledge workers to roughly infer whether a certain relationship among multiple indicators may exist. By zooming into a more detailed level, the users may play available videos and/or audios associated with an indicator in a particular country so as to obtain rich contextual information (Fig. 8).

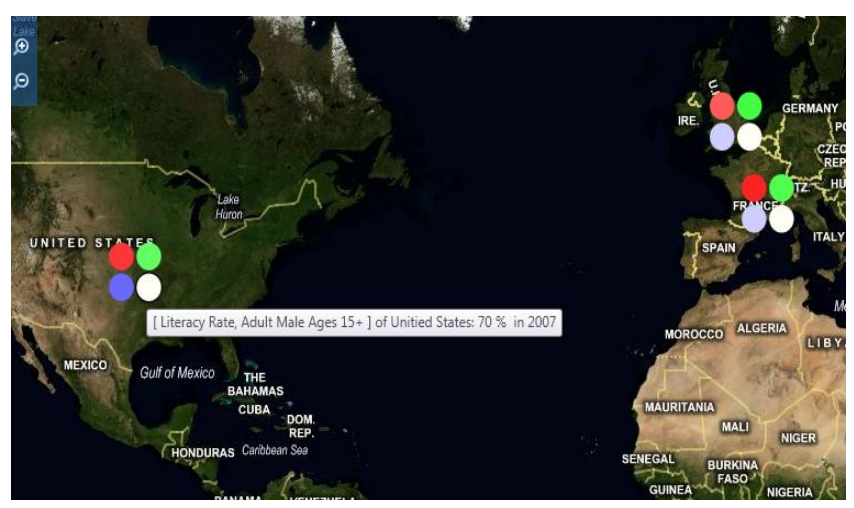

Fig. 6.Top layer: child related indicators by colours

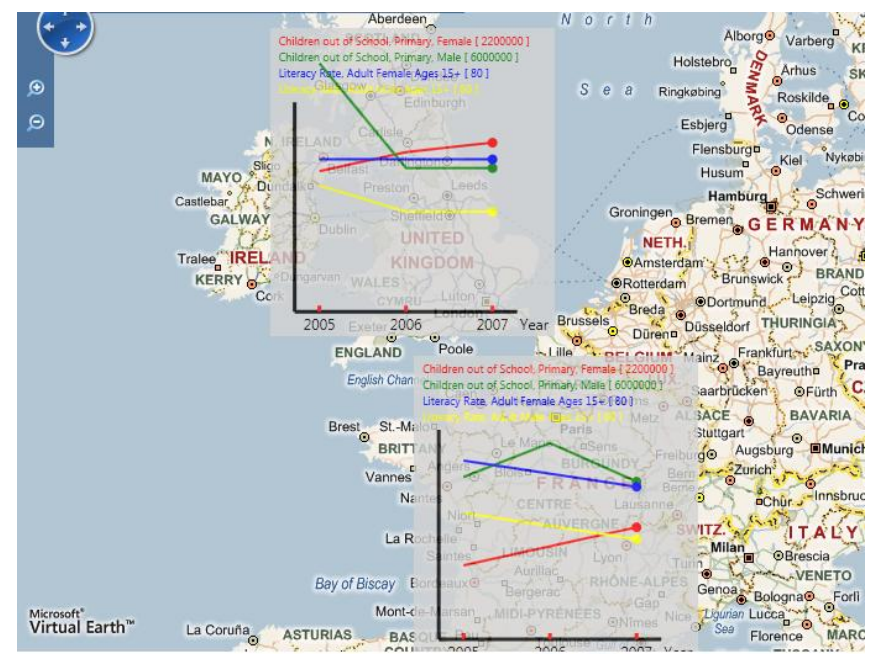

Fig. 7.Middle layer: showing trends of multiple Indicators by line graphs

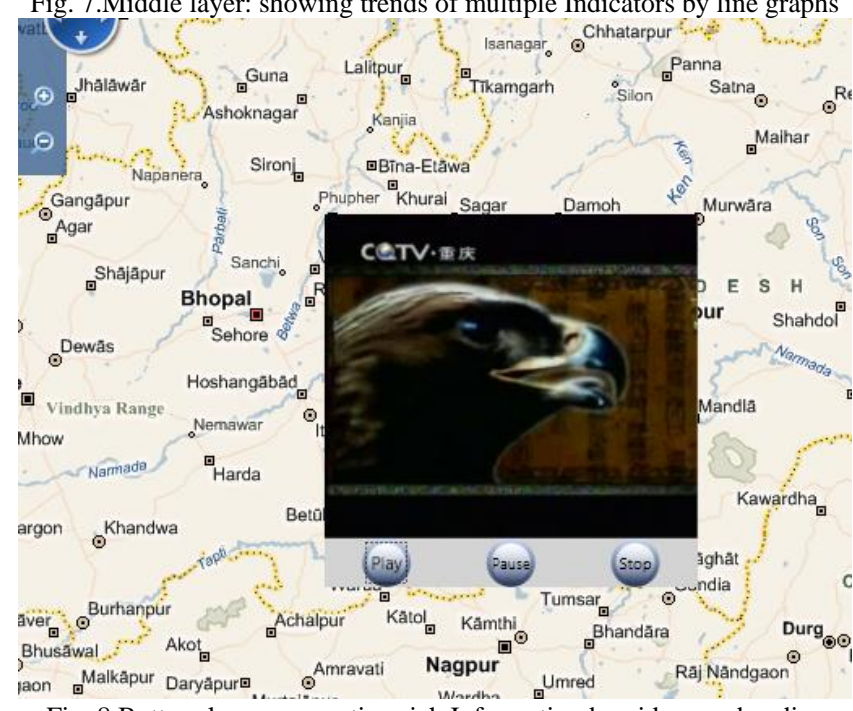

Fig. 8.Bottom layer: presenting rich Information by videos and audios

\section{CONCLUSION}

Knowledge visualization context is complicated and constantly changing in nature. Visualisations are integral for the creation, transfer and sharing of knowledge. The dynamic and changing problem and knowledge worker contexts often lead to changing visualization requirements that are ill-supported by existing knowledge management systems. One major challenge brought by the contextual complexity is 
how to enable knowledge workers to flexibly adapt knowledge visualizations to the changing and evolving context and maintain their effectiveness over time and space. To aid in addressing this challenge, we introduced concepts and model of contextual adaptive knowledge visualization environment and validated these artefacts through a concrete implementation. The CAVE prototype is demonstrated through the problem-visualization scenarios created for supporting changing contexts. It deserves to be pointed out that the current prototype has only been tested against a limited number of knowledge visualization context changes. Identifying/categorizing representative contextual changes and exploring/improving the support offered by the CAVE prototype will be accomplished in our future research.

\section{REFERENCES}

[1] R. A. Burkhard, "Learning from Architects: The Difference between Knowledge Visualization and Information Visualization," Proceedings of the 8th International Conference on Information Visualization, 2004, pp.519-524.

[2] R. Burkhard \& M. Meier, "Tube Map Visualization: Evaluation of a Novel Knowledge Visualization Application for the Transfer of Knowledge in Long-Term Projects," Journal of Universal Computer Science, vol.11, no.4, 2005, pp.473-494.

[3] A. Cañas, R. Carff, G. Hill, M. Carvalho, M. Arguedas, T. Eskridge, J. Lott, \& R. Carvajal, "Concept Maps: Integrating Knowledge and Information Visualization," Lecture Notes in Computer Science, in S.O. Tergan, \& T. Keller (eds.) Knowledge and Information Visualization: Searching for Synergies. Heidelberg: Springer-Verlag, vol. 3426, 2005, pp. 205-219.

[4] B. Pinaud, P. Kuntz, F. Guillet, \& V. Philippé, "Graphical Visualization in the Knowledge Management System Atanor,” Journal of Universal Computer Science, 2006, pp. 481-488.

[5] M.J. Eppler, A. Remo, \& R. Burkhard, "Visual Representations in Knowledge Management: Framework and Cases," Journal of Knowledge Management, vol. 11, no. 4, 2007, pp. 112 - 122.

[6] S. Bresciani \& M.J. Eppler, "Beyond Knowledge Visualization Usability: Toward a better Understanding of Business Diagram Adoption," Proceedings of the 13th International Conference on Information Visualization (IV09), Barcelona, 2009, pp. 15-17.

[7] S. Bresciani \& M.J. Eppler, "Choosing Knowledge Visualizations to Augment Cognition: the Managers' View," IEEE Proceedings of the International Conference on Information Visualization, London, 2010.

[8] M.J. Eppler \& R.A. Burkhard, "Knowledge Visualization," In D. Schwartz \& D. Te'eni (eds.), Encyclopedia of Knowledge Management, 2nd ed, 2011, pp. 987-999.

[9] A.K. Dey, "Understanding and Using Context," Personal and Ubiquitous Computing, vol. 5, no. 1, 2001, pp. 4-7.

[10] A. Schmidt, K.A. Aidoo, A. Takaluoma, U. Tuomela, K. Van Laerhoven, \& W. Van de Velde, "Advanced Interaction in Context," Proceedings of 1st International Symposium on Handheld and Ubiquitous Computing, Karlsruhe, Germany, 1999, pp. 89-101.

[11] G. Chen \& D. Kotz, A Survey of Context-Aware Mobile Computing Research, Technical Report, Department of Computer Science, Dartmouth College, 2000.

[12] V. Dhar \& R. Stein, Intelligent Decision Support Methods: the Science of Knowledge Work, Prentice Hall, Upper Saddle River, NJ, 1997.
[13] S.K. Card, J.D. Mackinlay, \& B. Shneiderman, Readings in Information Visualization: Using Vision to Think, Morgan Kaufman Publishers, San Francisco, California, 1999.

[14] S.K. Card \& J. Mackinlay, "The Structure of the Information Visualization Design Space," Proceedings of IEEE Symposium on Information Visualization, 1997, pp. 92-99.

[15] E.H. Chi \& J.T. Riedl, "An Operator Interaction Framework for Visualization Systems," Proceedings of IEEE Symposium on Information Visualization, 1998, pp. 63-70.

[16] S.D. Santos \& K. Brodlie, "Gaining Understanding of Multivariate and Multidimensional Data through Visualization," Computers and Graphics, vol.28, no.3, 2004, pp.311-325.

[17] NAG IRIS Explorer, Website, http://www.nag.co.uk, 2003

[18] W. Schroeder, K. Martin, \& B. Lorensen, The Visualization Toolkit an Object-Oriented Approach to 3D Graphics, 3rd Edition, Kitware Inc., 2003.

[19] R.B. Haber \& D.A. McNabb, Visualization Idioms: a Conceptual Model for Scientific Visualization Systems, in G.M. Nielson, B. Shriver, \& L.J. Rosenblum (eds.) Visualization in Scientific Computing, Silver Spring, MD: IEEE Computer Society Press, 1990, pp. 74-93.

[20] J. Heer \& M. Agrawala, "Software Design Patterns for Information Visualization," IEEE Transactions on Visualization and Computer Graphics, vol. 12, no. 5, 2006, pp. 853-860.

[21] E.H. Chi, P. Barry, J. Riedl, \& J. Konstan, "A Spreadsheet Approach to Information Visualization," Proceedings of IEEE Symposium on Information Visualization, 1997, pp. 17-24.

[22] B. Hibbard, "Top Ten Visualization Problems," ACM SIGGRAPH Computer Graphics, vol. 33, no. 2, 1999, pp. 21-22.

[23] E.R. Tufte, Beautiful Evidence, Graphics Press, Cheshire, 2006

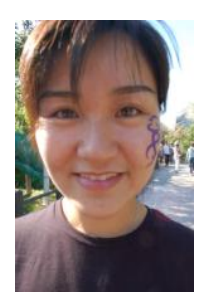

Xiaoyan Bai is a PhD student in Information Systems and Operations Management at the University of Auckland. She is passionate about visualization, knowledge management, adaptive systems, and decision support systems.

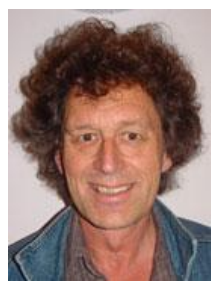

David White, who has 40 years experience in information design, is a lecturer in information systems and architect of Cecil Learning Management System at the University of Auckland.

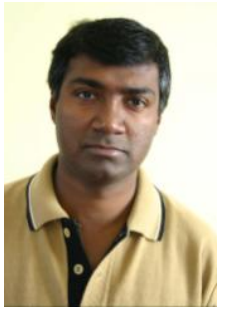

David Sundaram is Associate Professor in Information Systems and Operations Management at the University of Auckland. $\mathrm{He}$ is an engineer by background, a teacher, researcher, and consultant by profession, and a lifelong student. He is passionate about the modelling, design, and implementation of flexible and evolvable information, visualization, decision, knowledge, and social systems. 